\title{
Pandemic Influenza Plans in Residential Care Facilities
}

\author{
Hillary D. Lum, MD, PhD, ${ }^{a, b}$ Lona Mody, MD, MSc, ${ }^{c, d}$ Cari R. Levy, MD, PhD, ${ }^{b, e}$ and \\ Adit A. Ginde, MD, MPH ${ }^{f}$
}

OBJECTIVES: To identify characteristics of residential care facilities (RCFs) associated with having a pandemic influenza plan.

DESIGN: Nationally representative, cross-sectional survey.

SETTING: RCFs in the United States.

PARTICIPANTS: Participating facilities in the 2010 National Survey of RCFs $(\mathrm{N}=2,294)$, representing 31,030 assisted living facilities and personal care homes.

MEASUREMENTS: Facility-level characteristics associated with a pandemic influenza plan, including general organization descriptors, staffing, resident services, and immunization practices.

RESULTS: Forty-five percent $(95 \%$ confidence interval $(\mathrm{CI})=43-47 \%)$ had a pandemic plan, $14 \% \quad(95 \%$ $\mathrm{CI}=13-16 \%)$ had a plan in preparation, and $41 \%(95 \%$ $\mathrm{CI}=38-43 \%)$ had no plan. In the multivariable model, organization characteristics, staffing, and immunization practices were independently associated with the presence of a pandemic preparedness plan. Organization characteristics were larger size (extra large, $\mathrm{OR}=3.27,95 \%$ $\mathrm{CI}=1.96-5.46 ;$ large $, \mathrm{OR}=2.60,95 \% \mathrm{CI}=1.81-3.75$; medium, $\mathrm{OR}=1.66,95 \% \mathrm{CI}=1.21-2.27$ vs small), notfor-profit status $(\mathrm{OR}=1.65,95 \% \quad \mathrm{CI}=1.31-2.09 \quad$ vs for profit), and chain affiliation $(\mathrm{OR}=1.65,95 \%$ $\mathrm{CI}=1.31-2.09$ vs nonaffiliated). Staffing characteristics included number of registered nurse hours $(<15$ minutes, $\mathrm{OR}=1.36, \quad 95 \% \quad \mathrm{CI}=1.07-1.74$ vs no hours), any licensed practical nurse hours $(\mathrm{OR}=1.47,95 \%$ $\mathrm{CI}=1.08-1.99$ vs no hours), and at least 75 hours of required training for aides $(\mathrm{OR}=1.34,95 \% \mathrm{CI}=1.05$ 1.71 vs $<75$ hours). RCFs with high staff influenza

From the ${ }^{a}$ Division of Geriatric Medicine, School of Medicine, University

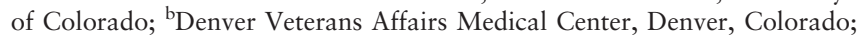
${ }^{c}$ Division of Geriatric and Palliative Care Medicine, University of

Michigan, ${ }^{\mathrm{d}}$ Geriatric Research Education and Clinical Center, Veteran

Affairs Ann Arbor Healthcare System, Ann Arbor, Michigan; ${ }^{\mathrm{e}}$ Division of Health Care Policy and Research, School of Medicine, University of

Colorado, and ${ }^{\mathrm{f}}$ Department of Emergency Medicine, School of Medicine, University of Colorado, Aurora, Colorado.

Address correspondence to Hillary D. Lum, Division of Geriatric Medicine, University of Colorado School of Medicine, 12631 E. 17th Ave, B-179, Aurora, CO 80045. E-mail: hillary.lum@ucdenver.edu

DOI: $10.1111 /$ jgs. 12879 vaccination rates $(81-100 \%, \mathrm{OR}=2.12,95 \% \mathrm{CI}=1.27-$ 3.53 vs $0 \%$ vaccinated) were also more likely to have a pandemic plan.

CONCLUSION: A majority of RCFs lacked a pandemic influenza plan. These facilities were smaller, for-profit, non-chain-affiliated RCFs and had lower staff vaccination rates. These characteristics may help target facilities that need to develop plans to handle a pandemic, or other disasters. J Am Geriatr Soc 62:1310-1316, 2014.

Key words: residential facility; assisted living facility; geriatric; pandemic; influenza

$\mathrm{F}$ our influenza pandemics in the 20th century caused millions of deaths, social disruption, and enormous economic consequences worldwide. ${ }^{1}$ According to the Department of Health and Human Services (HHS), when a pandemic strain emerges, $25 \%$ to $35 \%$ ( $\sim 75-105$ million people) of the U.S. population could develop the disease, and a significant proportion, particularly frail elderly adults, could die. ${ }^{2}$ Given concerns regarding whether society and the healthcare infrastructure can effectively handle the next influenza pandemic, ${ }^{3}$ prepandemic planning by healthcare facilities, especially long-term facilities that care for frail elderly adults, is critical to providing quality, uninterrupted care and limiting further spread of the influenza virus. ${ }^{4}$ Current pandemic preparedness efforts face numerous challenges, including inadequate supply of antiviral medications, a healthcare system that has not been designed to accommodate even a modest pandemic, and most worrisome, fragmented regional pandemic planning. ${ }^{3,5}$

Vulnerable older adults living in long-term facilities face unique challenges and will be at high risk from an influenza pandemic because of their advanced age and multiple chronic conditions. In addition to an estimated 1.5 million nursing home residents, ${ }^{6}$ there are also approximately 733,000 vulnerable residents of residential care facilities (RCFs). ${ }^{7}$ RCFs are a heterogeneous group of state-regulated facilities, such as assisted living facilities, personal care homes, and other residences, that serve an adult population by offering a range of personal care (e.g., bathing, dressing) or health-related services (e.g., medication assistance), room and board with at least two meals a day, and on-site 
supervision. ${ }^{8,9}$ RCFs are highly susceptible to virus outbreaks and rapid propagation in a pandemic. ${ }^{10}$ Unlike nursing homes, RCFs are not federally regulated, and infection prevention and control standards vary widely based on individual state regulations. ${ }^{11}$ If community- or state-level pandemic planning exists, RCFs are rarely included in these planning efforts. ${ }^{12}$ Nationally, the extent of influenza pandemic preparedness in RCFs is unknown, and research in this area is absent. ${ }^{4}$

The 2010 National Survey of Residential Care Facilities (NSRCF) presents a unique opportunity to provide a contemporary description of the prevalence of pandemic preparedness in U.S. long-term facilities that are not nursing homes. The goal of this study was to identify facility-level characteristics of RCFs associated with having a pandemic influenza plan. It was hypothesized that there would be specific characteristics related to facility organization, staffing, resident services, and immunization practices that would be independently associated with facility pandemic preparedness and might help target RCFs for improvement in disaster planning.

\section{METHODS}

\section{Study Design and Participants}

A secondary analysis of the facility-level data from the NSRCF, a nationally representative, cross-sectional survey of U.S. RCFs was performed. The study received a waiver from the Colorado Multiple institutional review board as an exempt study. Full details of survey methodology, including sampling, questionnaire, and interview processes, are described elsewhere. ${ }^{8}$ Briefly, in contrast to nursing homes, facilities included in this survey are RCFs, assisted living residences, board-and-care homes, congregate care programs, enriched housing programs, homes for the aged, personal care homes, and shared housing establishments that are licensed or otherwise regulated by a state and have four or more beds. Facilities licensed solely to serve mentally ill or developmentally disabled populations and nursing homes were excluded unless there was a specific unit meeting the eligibility requirements.

The NSRCF collected facility characteristics through in-person interviews with facility directors or designated staff. Facility-level data were collected on general organization, staffing, resident services and needs, and facility immunization practices. The primary sampling strata of facilities were defined according to number of beds and census regions, and within these sampling strata, 3,650 facilities were systematically and randomly sampled with probability proportional to size. ${ }^{8}$ Of the 2,302 RCFs that participated in the survey, the current analysis included 2,294 RCFs that responded to the primary outcome (pandemic influenza preparedness), which represent 31,030 RCFs nationally.

\section{Definition of Study Variables}

The primary outcome was pandemic influenza preparedness based on the question, "Has this facility developed a written plan for management of residents during an influenza pandemic?" Response options were yes, completed; yes, in progress; or no, not started. Independent variables were selected from the facility questionnaire based on potential association with pandemic preparedness factors, including general organization characteristics, staffing, available resident services, indicators of resident needs, and facility immunization practices. ${ }^{13,14}$

General organization characteristics included facility size, occupancy rate, ownership type, chain-affiliated (owned by chain or multifacility system), percentage of residents with services paid by Medicaid, duration of operation as a RCF, and location in a metropolitan statistical area. Staffing characteristics included whether the administrator or director had a certificate or license for managing facilities for older people, registered nurse (RN) direct care hours per resident per day, licensed practical nurse (LPN) direct care hours per resident per day, personal care aide direct care hours per resident per day, and hours of required formal training for personal care aides. Resident services variables included a designated unit for residents with dementia or Alzheimer's disease and percentage of single-occupancy living quarters. Resident needs variables included whether the RCF admission policy allowed admission of a resident who needs skilled nursing care on a regular basis, has moderate or severe cognitive impairment (i.e., the resident does not know who they are), or is unable to leave the facility in an emergency without help and also the percentage of residents who are confined to a bed or chair because of health problems. Positive responses included "yes" and "no specific policy-decisions on a case-by-case basis" for each admission policy. Facility vaccination practices included the rate of staff influenza vaccination, resident vaccination programs, and staff vaccination policy.

\section{Statistical Analysis}

Statistical analyses were performed using Stata 12.1 (StataCorp, College Station, TX). Using survey commands, the recommended stratified weights for the facility data were applied to accurately represent national estimates for RCFs. ${ }^{8}$ All results are presented as weighted values. All $P$-values were two-tailed, with $P<.05$ considered statistically significant. For the primary analysis, weighted proportions with $95 \%$ confidence intervals (CIs) were calculated for the selected covariates, according to whether a pandemic influenza plan existed or was in preparation or whether there was no plan. Independent variables were categorized based on clinically meaningful thresholds and to create relatively equal group sizes. Unadjusted, bivariate associations between characteristics and pandemic preparedness outcomes were determined using weighted chi-square tests. Multivariable logistic regression models were used to identify facility-level characteristics independently associated with RCF pandemic preparedness. The pandemic preparedness outcome was analyzed two ways: existing plan and plan in preparation or existing plan only. The analysis based on the presence of an existing plan only, which excluded $14 \%$ of RCFs that had a plan in progress but did not have a completed plan, was conducted as a more-stringent sensitivity analysis. The multivariable models included general organization characteristics, staffing characteristics, resident services, resident needs, and staff vaccination rate. 


\section{RESULTS}

Characteristics of the NSRCF facilities, stratified according to level of pandemic preparedness (existing plan, plan in preparation, no plan), are summarized in Table 1. Fortyfive percent of RCFs had an existing pandemic plan, $14 \%$ had a plan in preparation, and $41 \%$ did not have a plan. In unadjusted, bivariate analyses, being larger in size, nonprofit or government owned, chain affiliated, or operating as an RCF for more than 10 years were general organizational characteristics associated with being more likely to have a pandemic plan. RCFs with fewer than $50 \%$ of residents with Medicaid as the payment source were more likely to have a plan. Several staffing characteristics were associated with pandemic preparedness, including more direct patient care time for RNs, LPNs, and personal care aides and requiring more training for personal care aides. Higher staff influenza vaccination rates were also associated with pandemic preparedness.

In the multivariable model with the combined pandemic preparedness outcome of an existing plan or a plan in preparation (Table 2), several facility characteristics were independently associated with likelihood of having a pandemic plan: larger size, not-for-profit status, chain affiliated, some RN direct care hours per patient, any LPN direct care hours per patient, requiring at least 75 hours of training for aides, and higher staff influenza vaccination rates. RCFs with at least $50 \%$ Medicaid residents or at least $10 \%$ of residents confined to bed or chair were less likely to have the combined pandemic plan outcome.

As a sensitivity analysis, the RCF characteristics associated with only having an existing plan was also tested, thus excluding the $14 \%$ of RCFs that had a plan in progress. In this more-stringent model of pandemic preparedness (Table 2), two additional characteristics were associated with being less likely to have a completed pandemic plan: having at least some single-occupancy rooms and ability to admit residents with skilled nursing needs. Although the presence of a designated dementia unit was associated with pandemic preparedness in the unadjusted analysis (Table $1, P<.001$ ), this resident service characteristic was associated only with the more stringent "existing plan only" outcome multivariable model (Table 2). Overall, when comparing the multivariable models, the six facility characteristics that were independently associated with pandemic preparedness in both models were larger size, nonprofit or government ownership, chain affiliation, having less than 15 minutes of $\mathrm{RN}$ direct care time (vs none or more time), having any LPN direct care time, and higher staff vaccination rates.

Facility staff and resident vaccination practices were analyzed to better understand current seasonal influenza vaccination practices. The most common resident-level vaccination program in surveyed RCFs was "personal physician order for each resident" (54\%), followed by "facility-wide standing orders" (19\%). As shown in Table 3, the presence of a policy to promote resident influenza vaccination was associated with pandemic preparedness in bivariate analyses. Strategies to increase staff influenza vaccination rates were also associated with pandemic preparedness.

\section{DISCUSSION}

This study provides the first national estimate of pandemic preparedness in U.S. RCFs. Specifically, in 2010, 54\% RCFs lacked a complete pandemic influenza plan. RCFs that did not have a plan were more likely to be smaller, for-profit, non-chain-affiliated RCFs or have lower staff vaccination rates. The multivariable analysis also found that RCFs with the highest proportions of Medicaid residents or residents confined to bed or chair, both indicators of highly vulnerable residents, were less likely to have conducted any prepandemic planning. Thus, despite greater resident vulnerability, some RCFs appeared to be unable to prioritize resources for pandemic preparedness. Given that it has been suggested that pandemic influenza plans are important in reducing the risks of a potentially devastating influenza outbreak among frail elderly adults in residential care settings, the identification of facility-level characteristics that are independently associated with pandemic preparedness in RCFs may help long-term care administrators and providers, consumer advocates, and policy-makers target facilities that still need to develop plans to handle a pandemic, as well as other natural or manmade disasters. ${ }^{4}$

This national, cross-sectional analysis reports a contemporary prevalence of pandemic preparedness in long-term facilities other than nursing homes. Before this study, the only evaluation of RCF pandemic preparedness was in Nebraska assisted living facilities, where approximately one-third of assisted living facilities had a pandemic influenza plan. ${ }^{15}$ Because RCFs are regulated at the state level, pandemic preparedness in RCFs may vary significantly. Even though nursing homes are federally regulated, the extent of influenza pandemic preparedness in U.S. nursing homes remains unknown. To the knowledge of the authors, the only report of pandemic preparedness in nursing homes is a survey of Michigan and Nebraska nursing homes, which found that $23 \%$ of respondents had taken undertaken some pandemic influenza preparedness planning, whereas $52 \%$ did not have a plan. ${ }^{13}$ The low rates of pandemic preparedness in nursing homes suggest that the presence of additional regulation may not be providing a clear impetus for pandemic preparedness.

The Centers for Disease Control and Prevention and HHS have developed a checklist to help long-term facilities assess and improve their preparedness for responding to pandemic influenza. ${ }^{16}$ The checklist recommends a structure for planning and decision-making, development of a written pandemic influenza plan, and several elements of the plan. Given the wide variation among RCFs, each facility will need to adapt the checklist to meet its unique characteristics. Because RCFs are not federally regulated, facilities that are developing a comprehensive pandemic influenza plan need to incorporate information from state, regional, and local health departments; emergency management agencies; and other organizations. ${ }^{17} \mathrm{RCF}$ s that have a pandemic influenza plan can conduct a self-assessment using a tool that evaluates the degree of preparedness and identifies problems related to preparedness in the longterm care setting. ${ }^{18}$ 
Table 1. Characteristics of Residential Care Facilities (RCFs) According to Status of Pandemic Influenza Preparedness

$\begin{array}{ccc}\begin{array}{c}\text { Existing Plan, } \\ n=1,175\end{array} & \text { Plan in Preparation, } & \text { No Plan, } \\ n=340 & n=79\end{array}$

\section{Characteristic}

All facilities $(N=2,294)$

General characteristic

Facility size (number of licensed beds)

Small (4-10)

Medium (11-25)

Large (26-100)

Extra large $(>100)$

Occupancy, \%

1-65

66-80

$81-95$

$>95$

Ownership type

Private, for profit

Nonprofit, non-federal government

Chain affiliated

Medicaid, \%

$0-19$

$20-49$

$\geq 50$

Operation as RCF for $\geq 10$ years

Yes

No

Unknown

Staffing characteristic

Administrator certified

Minutes of registered nurse direct care per patient day

0

$<15$

$\geq 15$

Minutes of licensed practical nurse direct care per patient day 0

$<15$

$>15$

Hours of personal care aide direct care per patient day ${ }^{b}$

$<2$
$>2$

$\geq 2$

$<75$

$\geq 75$

Staff vaccination rates, $\%$

0

$1-40$

$41-80$

Resident services characteristic

Designated dementia care unit available

Single-occupancy living quarters, \% 0

1- 49

50-99

100

Resident need characteristic

Skilled nursing needs

Moderate to severe cognitive impairment

Unable to leave in an emergency

Confined to bed or chair because of health problems
Metropolitan Statistical Area

$81-100$

Weighted Percentage (95\% Confidence Interval) ${ }^{a}$

$P$-Value

$45(43-47) \quad 14(13-16) \quad 41(38-43)$

\begin{tabular}{|c|c|c|c|}
\hline $29(26-33)$ & $14(11-17)$ & 57 (52-61) & $<.001$ \\
\hline $47(43-51)$ & 17 (14-20) & $36(32-40)$ & \\
\hline $64(61-67)$ & $14(12-16)$ & 22 (19-25) & \\
\hline 74 (67-79) & $13(9.2-19)$ & $13(9.1-18)$ & \\
\hline $43(38-48)$ & $15(12-19)$ & $42(37-47)$ & .24 \\
\hline $46(41-51)$ & $13(10-16)$ & $41(36-46)$ & \\
\hline $48(44-52)$ & $16(13-19)$ & $45(32-40)$ & \\
\hline $41(36-47)$ & $14(11-18)$ & $45(39-50)$ & \\
\hline $42(38-44)$ & $14(13-17)$ & $44(41-47)$ & $<.001$ \\
\hline 60 (55-65) & $14(11-18)$ & $26(21-30)$ & \\
\hline $59(55-63)$ & $14(12-17)$ & 27 (24-31) & $<.001$ \\
\hline $49(45-52)$ & $14(11-17)$ & $37(34-41)$ & $<.001$ \\
\hline $49(44-54)$ & $14(11-18)$ & $37(21-42)$ & \\
\hline 33 (29-38) & 15 (12-19) & $51(46-56)$ & \\
\hline $50(47-53)$ & $12(10-14)$ & 37 (34-41) & $<.001$ \\
\hline $42(40-45)$ & $14(12-16)$ & $44(41-47)$ & $<.001$ \\
\hline $45(40-49)$ & 17 (13-20) & 39 (34-44) & \\
\hline $74(67-79)$ & $13(9.2-19)$ & $13(9.1-18)$ & \\
\hline $45(42-47)$ & $14(12-16)$ & $41(39-44)$ & .68 \\
\hline $38(35-41)$ & $14(11-16)$ & $48(45-52)$ & $<.001$ \\
\hline 59 (55-63) & $15(12-17)$ & $26(23-30)$ & \\
\hline $43(36-51)$ & $19(13-26)$ & $38(30-47)$ & \\
\hline 36 (33-39) & $14(12-17)$ & $50(47-53)$ & $<.001$ \\
\hline 62 (58-67) & $15(12-18)$ & 23 (19-28) & \\
\hline $64(59-69)$ & $14(11-18)$ & $22(17-27)$ & \\
\hline $52(48-55)$ & $15(13-18)$ & $33(30-36)$ & $<.001$ \\
\hline $41(42-47)$ & $14(13-16)$ & $41(39-43)$ & \\
\hline $43(41-46)$ & $16(14-19)$ & $40(37-43)$ & .01 \\
\hline $52(47-56)$ & $12(9.4-15)$ & $36(32-41)$ & \\
\hline $5.4(3.9-7.5)$ & $5.3(2.8-9.8)$ & $18(15-22)$ & $<.001$ \\
\hline $18(16-21)$ & 21 (16-26) & $15(12-18)$ & \\
\hline $29(26-32)$ & $29(24-36)$ & $20(17-23)$ & \\
\hline $48(45-49)$ & $44(38-51)$ & 47 (43-51) & \\
\hline 64 (59-69) & $15(12-20)$ & $20(16-25)$ & $<.001$ \\
\hline 57 (54-61) & $16(14-19)$ & $26(23-30)$ & $<.001$ \\
\hline $38(33-43)$ & $15(11-19)$ & $47(42-53)$ & \\
\hline $40(36-45)$ & $13(10-17)$ & $47(42-52)$ & \\
\hline $37(31-44)$ & $13(9.0-18)$ & $50(43-57)$ & \\
\hline $37(32-42)$ & $11(8.1-14)$ & $52(47-57)$ & $<.001$ \\
\hline $43(40-46)$ & $15(12-17)$ & 42 (39-46) & .07 \\
\hline $44(41-46)$ & $14(12-16)$ & 42 (39-45) & .11 \\
\hline $34(29-38)$ & $17(13-21)$ & 49 (44-55) & $<.001$ \\
\hline
\end{tabular}

${ }^{a}$ Details for weighted analysis are available. ${ }^{8}$

${ }^{\mathrm{b}}$ Of facilities with personal care aides $(\mathrm{n}=2,197)$. 
Table 2. Multivariable Models of Pandemic Preparedness Outcomes

Existing Plan or Plan in Preparation, $n=1,515$ (59\%)
Existing Plan Only, $n=1,175(45 \%)$

\section{Residential Care \\ Facility Characteristic}

Odds Ratio

(95\% Confidence Interval)

\begin{tabular}{|c|c|c|}
\hline \multicolumn{3}{|l|}{ General characteristic } \\
\hline \multicolumn{3}{|l|}{ Facility size (number of licensed beds) (reference small (4-10)) } \\
\hline Large $(26-100)$ & $2.60(1.81-3.75)^{\mathrm{a}}$ & $2.04(1.39-2.98)^{\mathrm{a}}$ \\
\hline \multicolumn{3}{|l|}{ Occupancy, \% (reference 1-65) } \\
\hline $66-80$ & $1.16(0.83-1.62)$ & $0.96(0.67-1.38)$ \\
\hline $81-95$ & $0.91(0.66-1.26)$ & $0.91(0.64-1.30)$ \\
\hline Nonprofit, non-federal government & $1.49(1.13-1.97)^{\mathrm{a}}$ & $1.52(1.11-2.09)^{\mathrm{a}}$ \\
\hline Chain affiliated & $1.65(1.31-2.09)^{\mathrm{a}}$ & $1.58(1.22-2.06)^{\mathrm{a}}$ \\
\hline \multicolumn{3}{|l|}{ Medicaid, \% (reference 0) } \\
\hline $20-49$ & $0.96(0.73-1.25)$ & $0.99(0.73-1.33)$ \\
\hline$\geq 50$ & $0.73(0.54-0.98)^{a}$ & $0.77(0.56-1.04)$ \\
\hline Operation as residential care facility for $\geq 10$ years & $1.18(0.93-1.51)$ & $0.92(0.71-1.19)$ \\
\hline$<15$ & $1.36(1.07-1.74)^{\mathrm{a}}$ & $1.42(1.09-1.87)^{\mathrm{a}}$ \\
\hline$\geq 15$ & $1.16(0.80-1.68)$ & $1.41(0.93-2.14)$ \\
\hline \multicolumn{3}{|c|}{ Minutes of licensed practical nurse direct care per resident day (reference 0) } \\
\hline$<15$ & $1.33(1.01-1.74)^{\mathrm{a}}$ & $1.43(1.02-2.00)^{\mathrm{a}}$ \\
\hline$\geq 15$ & $1.47(1.08-1.99)^{\mathrm{a}}$ & $1.49(1.05-2.12)^{\mathrm{a}}$ \\
\hline \multicolumn{3}{|c|}{ Hours of personal care aide direct care per resident day (reference <2) } \\
\hline 0 & $0.81(0.26-2.47)$ & $0.72(0.25-2.11)$ \\
\hline$\geq 2$ & $1.29(0.99-1.69)$ & $1.07(0.80-1.43)$ \\
\hline$\geq 7 \overline{5}$ required training hours for aides (reference $<75$ hours) & $1.34(1.05-1.71)^{\mathrm{a}}$ & $1.09(0.83-1.42)$ \\
\hline \multicolumn{3}{|l|}{ Staff vaccination rate, \% (reference 0 ) } \\
\hline $1-40$ & $1.38(0.81-2.38)$ & $2.01(1.18-3.42)^{\mathrm{a}}$ \\
\hline $41-80$ & $1.67(0.99-2.82)$ & $2.52(1.51-4.21)^{\mathrm{a}}$ \\
\hline $81-100$ & $2.12(1.27-3.53)^{\mathrm{a}}$ & $2.52(1.54-4.11)^{\mathrm{a}}$ \\
\hline Moderate to severe cognitive impairment & $1.13(0.86-1.47)$ & $1.22(0.92-1.61)$ \\
\hline Unable to leave in an emergency & $0.97(0.75-1.24)$ & $0.96(0.73-1.26)$ \\
\hline Confined to bed or chair because of health problems & $0.71(0.52-0.95)^{\mathrm{a}}$ & $0.96(0.70-1.31)$ \\
\hline
\end{tabular}

${ }^{\mathrm{a} P}<.05$.

This analysis found that RCFs with higher staff vaccination rates were more likely to have a pandemic influenza plan. These data add to studies in long-term care settings regarding employee vaccination programs. The association between healthcare worker vaccination rates and patient "protection" from seasonal influenza infection has been reported. ${ }^{19}$ Not surprisingly, there was a strong association between employee vaccination rate and RCF pandemic preparedness, indicating that these facilities dedicate attention and resources to interventions intended to limit influenza morbidity. In addition to the protection of residents and staff, effective seasonal and pandemic preparedness may limit the spread of influenza infection to the broader local community that intersects with RCFs, such as family, visitors, indirect care workers, transporters, and suppliers.

This study has several limitations. The responses were based on facility self-report and not verified, which may have introduced bias or inaccuracy. The survey did not assess specific elements of pandemic preparedness plans, including whether the plans contain components of the HHS checklist. In addition, the NSRCF includes a heterogeneous group of facilities that serve populations with a broad range of needs. ${ }^{7,9}$ Although the analysis included general organization, staffing, resident services, resident 
Table 3. Resident and Staff Vaccination Programs According to Pandemic Preparedness

Existing Plan, $n=1,175(45 \%)$
Plan in Preparation, $n=340(14 \%)$
No Plan, $n=779(41 \%)$

Facility Vaccination Characteristic

Weighted Percentage (95\% Confidence Interval)

$P$-Value

\begin{tabular}{llllc}
\hline Resident vaccination program & & & & \\
$\quad$ Facility-wide standing orders & $51(46-56)$ & $19(15-24)$ & $30(25-35)$ & $<.001$ \\
Preprinted admission orders & $66(56-74)$ & $10(5.8-17)$ & $24(17-33)$ \\
Advance physician or nurse practitioner orders for all residents & $49(41-57)$ & $10(6.7-16)$ & $41(33-49)$ \\
Personal physician order for each resident & $44(40-47)$ & $14(12-17)$ & $42(39-46)$ \\
$\quad$ None of the above & $31(25-37)$ & $12(8.7-18)$ & $57(50-64)$ & \\
Staff vaccinations recommended & $48(45-51)$ & $15(13-17)$ & $37(34-40)$ & $<.001$ \\
Staff vaccinations offered on-site & $58(56-62)$ & $14(13-16)$ & $27(24-30)$ & $<.001$ \\
Staff vaccinations offered for free & $60(56-63)$ & $12(10-14)$ & $29(26-32)$ & $<.001$ \\
Staff vaccinations offered at reduced cost & $52(44-60)$ & $17(12-24)$ & $31(24-39)$ & .04 \\
Staff incentives for vaccinations & $67(56-76)$ & $10(5.5-19)$ & $22(14-33)$ & $<.001$ \\
Proof of vaccination required for work & $51(44-59)$ & $17(12-24)$ & $32(25-39)$ & .04 \\
Staff furlough or patient restriction policy & $62(57-68)$ & $12(8.8-16)$ & $26(21-32)$ & $<.001$ \\
\hline
\end{tabular}

needs, and immunization practices, it was not possible to account completely for the heterogeneity of RCFs and other potential factors that may be relevant to pandemic preparedness in RCFs, such as hospital affiliation; collaborations with state, community, or referring hospital pandemic preparedness programs; facility-based infection control programs; presence of an overall emergency or disaster response team; and experience with prior disasters. In particular, there was limited detail on the type of medical care available at different RCFs beyond direct healthcare worker staffing, whether residents with skilled nursing needs were admitted, and immunization policies. Thus, while even some RN or LPN availability per patient was associated with pandemic preparedness, more-specific detail on the type of care provided was not available. However, the NSRCF is a large and nationally representative survey that provides contemporary estimates and characteristics of pandemic preparedness.

In conclusion, more than half of U.S. RCFs lacked a plan to address an influenza pandemic, highlighting the need to support prepandemic planning in this expanding sector of the U.S. healthcare system. Facility-level characteristics, including smaller size, for-profit ownership, and lack of chain affiliation, were identified that were associated with the absence of a pandemic plan and could be used to target regional-level prepandemic planning efforts. Future research is needed to assess the quality of existing pandemic response plans in RCFs. Timely community, state, regional, and national pandemic preparedness that includes RCFs and nursing homes is critical to providing quality, uninterrupted care to frail elderly adults and preventing further spread of the influenza virus in this vulnerable population.

\section{ACKNOWLEDGMENTS}

Presented at the 2014 American Geriatrics Society Annual Scientific Meeting.

Conflict of Interest: The authors report that there are no financial, personal, or potential conflicts of interest.

Dr. Mody was supported by National Institute on Aging Grants R01 AG032298, R01 AG41780, and R18
HS019979 and University of Michigan Claude D. Pepper Older Americans Independence Center Grant P30 AG024824. Dr. Ginde was supported by National Institute on Aging Grant K23 AG040708.

Author Contributions: Drs. Lum and Ginde had full access to all of the data in the study and take responsibility for the integrity of the data and the accuracy of the data analysis. Study concept and design: Lum, Mody, Levy, Ginde. Data acquisition and statistical analysis: Ginde. Analysis and interpretation of data: Lum, Mody, Levy, Ginde. Preparation of the manuscript: Lum, Ginde. Critical revision of the manuscript for important intellectual content: Lum, Mody, Levy, Ginde.

Sponsor's Role: The sponsors had no role in the design, methods, analysis, or preparation of the manuscript. The views in this article are those of the authors and do not necessarily represent the views of the Department of Veterans Affairs.

\section{REFERENCES}

1. Lagacé-Wiens PRS, Rubinstein E, Gumel A. Influenza epidemiology-past, present, and future. Crit Care Med 2010;38(4 Suppl):e1-e9.

2. U.S. Department of Health and Human Services [on-line]. HHS Pandemic Influenza Plan. 2005:1-396. Available at http://www.flu.gov/planningpreparedness/federal/hhspandemicinfluenzaplan.pdf Accessed January 2, 2014.

3. Bartlett JG. Planning for avian influenza. Ann Intern Med 2006;145:141144.

4. Mody L, Cinti S. Pandemic influenza planning in nursing homes: Are we prepared? J Am Geriatr Soc 2007;55:1431-1437.

5. Iskander J, Strikas RA, Gensheimer KF et al. Pandemic influenza planning, United States, 1978-2008. Emerg Infect Dis 2013;19:879-885.

6. Jones AL, Dwyer LL, Bercovitz AR et al. The National Nursing Home Survey: 2004 overview. Vital Health Stat 13 2009;167:1-155.

7. Caffrey C, Sengupta M, Park-Lee E et al. Residents Living in Residential Care Facilities: United States, 2010. Hyattsville, MD: National Center for Health Statistics, 2012.

8. Moss AJ, Harris-Kojetin LD, Sengupta M et al. Design and operation of the 2010 National Survey of Residential Care Facilities. Vital Health Stat 1 2011;54:1-131.

9. Park-Lee E, Caffrey C, Sengupta M et al. Residential care facilities: A key sector in the spectrum of long-term care providers in the United States. NCHS Data Brief 2011;12:1-8.

10. Nuno M, Reichert T, Chowell G et al. Protecting residential care facilities. PNAS 2008;105:10625-10630. 
11. Kossover RA, Chi CJ, Wise ME et al. Infection prevention and control standards in assisted living facilities: Are residents' needs being met? J Am Med Dir Assoc 2014;15:47-53.

12. National Association of County and City Health Officials. 2010 National profile of local health departments. 2011 [on-line]. Available at http:// www.naccho.org/topics/infrastructure/profile/resources/2010report/upload/ 2010_Profile_main_report-web.pdf Accessed January 2, 2014.

13. Smith PW, Shostrom V, Smith A et al. Preparedness for pandemic influenza in nursing homes: A 2-state survey. JAMA 2008;300:392-394.

14. Centers for Disease Control Home [on-line]. Survey Methodology, Documentation, and Data Files. 2013. Available at http://www.cdc.gov/nchs/ nsrcf/nsrcf_questionnaires.htm Accessed December 2, 2013.

15. Smith PW, Smith AW, Meza JL. Influenza preparedness in Nebraska assisted living facilities. Biosecur Bioterror 2009;7:429-432.
16. Centers for Disease Control Home [on-line]. Long-term care and other residential facilities pandemic influenza planning checklist. 2006. Available at http://www.flu.gov/planningpreparedness/hospital/longtermcarechecklist. html Accessed September 14, 2013.

17. Volkman JC, Rebmann T, Hilley S et al. Infection prevention disaster preparedness planning for long-term care facilities. Am J Infect Control 2012;40:206-210.

18. Smith PW, Hansen K, Sayles $\mathrm{H}$ et al. A long-term care facility pandemic influenza preparedness planning assessment tool. Am J Disaster Med 2011;6:219-230.

19. Salgado CD, Giannetta ET, Hayden FG et al. Preventing nosocomial influenza by improving the vaccine acceptance rate of clinicians. Infect Control Hosp Epidemiol 2004;25:923-928. 